\title{
Allometric equations to estimate the above-ground biomass of trees in the tropical secondary forests of different ages
}

\author{
KARYATI $^{1, \boldsymbol{v}}$, ISA B. IPOR ${ }^{2}$, ISMAIL JUSOH ${ }^{2}$, MOHD. EFFENDI WASLI ${ }^{2}$ \\ ${ }^{1}$ Faculty of Forestry, University of Mulawarman. Jl. Ki Hajar Dewantara, P.O. Box 1013, Samarinda 75119, East Kalimantan, Indonesia. \\ Tel.: +62-541-735089, 749068, Fax.: +62-541-735379, `email: karyati@fahutan.unmul.ac.id; karyati.hanapi@yahoo.com \\ ${ }^{2}$ Faculty of Resource Science and Technology, Universiti Malaysia Sarawak. 94300 Kota Samarahan, Sarawak, Malaysia
}

Manuscript received: 28 June 2019. Revision accepted: 1 August 2019.

\begin{abstract}
Karyati, Ipor IB, Jusoh I, Wasli ME. 2019. Allometric equations to estimate the above-ground biomass of trees in the tropical secondary forests of different ages. Biodiversitas 20: 2427-2436. The allometric equations for trees of secondary forests of different ages in abandoned lands after shifting cultivation are still rarely available. The objective of this study was to develop allometric equations to estimate the above-ground biomass (AGB) of trees (DBH of $\geq 5 \mathrm{~cm}$ ) in the tropical secondary forest of different ages, namely 5, 10, and 20 years after abandonment. The selected trees in this study represented the dominant and rare species and DBH classes in each study site. The trunk dry biomass and AGB showed strong correlations (adjusted $\mathrm{R}^{2}=0.59-0.95$ ) with diameter at breast height (DBH) and height. The leaf and branch dry biomass had weak correlations with height (adjusted $\mathrm{R}^{2}=0.36-0.50$ ). The developed allometric equations were suitable for trees of secondary forests of different ages, because the selected samples used in the destructive method were based on a field inventory data of forest structure and floristic composition.
\end{abstract}

Keywords: Allometric equation, biomass, destructive method, secondary forest

\section{INTRODUCTION}

There are vast areas of swidden fallow secondary forests in the tropics and it is important to measure the regrowth rates of these forests and to estimate their potentials as carbon sinks (Hashimotio et al. 2000). Tree species richness and dominance are important factors to consider in estimating tree carbon storage in hyperdiverse forests (Ruiz-Jaen and Potvin 2010). Like any natural forest ecosystem, secondary forests provide both tangible and intangible goods and services. Secondary forests contribute to the sequestration of carbon, the conservation of biodiversity and the protection of soil, especially in the recovery of soil fertility after cultivation (Perera 2001). The total standing above-ground biomass (AGB) of woody vegetation is often one of the largest carbon pools. The AGB comprises all woody stems, branches, and leaves of living trees, creepers, climbers, epiphytes, and herbaceous undergrowth (Hairiah et al. 2001). The estimation of AGB is an essential aspect of studies of carbon stocks as well as the effects of deforestation and carbon sequestration on the global carbon balance (Ketterings et al. 2001). Brower et al. (1990) stated that because direct measurement of biomass cannot be made on the entire community or population, samples must be taken from a community or population. Ketterings et al. (2001) pointed out that weighing tree biomass in the field is undoubtedly the most accurate method of estimating AGB, but it is extremely time-consuming and destructive, generally limited to small areas and small tree sample sizes.

An estimate of the vegetation biomass can provide information about the nutrients and carbon stored in the vegetation as a whole, or the amount in specific fractions such as extractable wood (Hairiah et al. 2001). Allometry is an effective method for accurately estimating biomass of trees, tree components and stands. The labor and expense of constructing and validating the necessary equations limit the application of the allometric approach in biomass sampling (MacDicken 1997). It is hardly ever possible to measure all biomass on a sufficiently large sample area by destructive methods and some allometric equations are used to estimate the biomass of individual trees based on an easily measured property such as their trunk diameter (Hairiah et al. 2001). Various dimensions and partial biomass of trees, such as component parts of bole wood, bark, branch, and foliage mass are estimated from diameter at breast height $(\mathrm{DBH})$ by allometric correlation method (Basuki et al. 2009; Curtis 2008).

Allometric equation is regression expressing the relationship between the dimension of a tree or different parts of plants with the biomass (Heriansyah et al. 2002; Ministry of Forestry Indonesia 2011). Regression models are used to convert inventory data into an estimate of the biomass of trees (Chave et al. 2005; Ministry of Forestry Indonesia 2011). Once an allometric equation has been established for different classes of trees in a vegetation type, one only needs to measure the $\mathrm{DBH}$ or other parameters, such as height, used as a basis for equation to estimate the biomass of individual trees and total biomass or carbon content (Hairiah et al. 2001; Heriansyah et al. 2002). To measure the biomass of vegetation that includes trees is not easy, especially in mixed, uneven-aged stands. It requires considerable labor and it is difficult to obtain an accurate measurement given the variability of tree size 
distribution (Hairiah et al. 2001).

The allometric equations for trees in swidden fallow secondary forests of different ages, such as 5,10 , and 20 year-fallow periods are still rarely available. Several previous developed allometric equations are mainly for trees of primary rain forests (Basuki et al. 2009; Brown 1997; Chamber et al. 2001; Chave et al. 2005; Kawahara et al. 1981; Rai and Proctor 1986; Yamakura et al. 1986), while several allometric equations for trees of secondary forests were reported by Hashimoto et al. (2004), Kenzo et al. 2009a, Kenzo et al. (2009b), Ketterings et al. (2001), Kiyono and Hastaniah (2005); Nelson et al. (1999) and Sierra et al. (2007). When no specific allometric equations to estimate AGB at different age secondary forests is available, these proposed equations may be used to estimate AGB at different stages of fallow periods. Data on the structure, floristic composition, and diversity of the secondary forests of different ages are needed to estimate their AGB and carbon sequestration. Because it is crucial to accurately estimate AGB in secondary forests of different ages, the suitable allometric equations need to be developed. This study was conducted to develop allometric equations for accurate estimation of AGB at the different stages of fallows.

\section{MATERIALS AND METHODS}

\section{Study sites}

The study was carried out in 5-, 10-, and 20-year-old secondary forest in Sarawak, East Malaysia, respectively located in $01^{\circ} 04^{\prime} 43.3^{\prime \prime} \mathrm{N} \quad 110^{\circ} 59^{\prime} 02.0^{\prime \prime} \mathrm{E}, \quad 01^{\circ} 03^{\prime} 55.9^{\prime \prime} \mathrm{N}$ $110^{\circ} 55^{\prime} 51.4^{\prime \prime} \mathrm{E}$, and $01^{\circ} 03^{\prime} 59.3^{\prime \prime} \mathrm{N} 110^{\circ} 53^{\prime} 34.4^{\prime \prime} \mathrm{E}$. The previous study on composition and diversity of trees (Karyati et al. 2018) as well as their soil properties (Karyati et al. 2014) has been done in these study sites. The forest type in the study plots was lowland mixed dipterocarp forest with heath forest (kerangas) (Kendawang et al. 2007). The soil was dominated by acidic soil $\left(\mathrm{pH}\left(\mathrm{H}_{2} \mathrm{O}\right)<\right.$ 5) having low content of T-C, T-N and exchangeable bases (Karyati et al. 2014).

\section{Procedures}

\section{Selecting sample trees}

A total number of 30 trees (DBH of $\geq 5 \mathrm{~cm}$ ) were selected in each age class $(5,10$, and 20 years old) of secondary forests, with consideration of the species and DBH, not considering individuals with damaged crowns or broken trunks. Almost $90 \%$ of the selected trees were categorized as the dominant species in terms of density and Importance Value Index (IVi) in each study site as reported by Karyati et al. (2018), while few selected trees represented the rare species. The DBH of selected trees proportionally represented each DBH class in each study site.

\section{Biomass measurements}

The standing DBH $(1.3 \mathrm{~m})$ of selected trees were measured using diameter tapes. Measurement of the total height of the sample trees was completed once the trees had been felled. The harvested trees were divided into several factions, each of which was 1 meter-long. After that, parts of the trees were separated into leaves and twigs (hereafter called leaves), branches, and trunks in the field. All fractions were weighed at the field in fresh condition. The scale used depended on the estimated weight of the fraction to be weighed. Three or four disk samples of trunk of 2-5 $\mathrm{cm}$ thick were taken by cutting a cross-section of the trunk with a minimum size of a quarter of the trunk circumference. Three disk samples were collected from the harvested trees with less than 10 fractions and 4 disk samples were collected for the harvested trees with $\geq 10$ fractions. Five branch samples were taken by cutting a cross-section of the branch, $20-30 \mathrm{~cm}$ in length, from each sample tree. Five leaf and twig samples, 100-300 grams in weight, were taken from each sample tree.

\section{Data analyses}

The wood density (WD) of each disk sample was determined using the formula below (Bowyer et al. 2003; Chave 2006; Marklund 1986):

$$
\mathrm{WD}=\mathrm{dw} / \mathrm{V}
$$

Where: WD = wood basic density $\left(\mathrm{g} \mathrm{cm}^{-3}\right) ; \mathrm{dw}=$ oven dry weight $(\mathrm{g}) ; \mathrm{V}=$ saturated volume $\left(\mathrm{cm}^{3}\right)$.

The total oven-dry weight of each tree part was determined using the following formula (Hairiah et al. 2001; Hairiah and Rahayu 2007; Ministry of Forestry Indonesia 2011):

$$
\mathrm{dw}=(\mathrm{sdw} \times \mathrm{fw}) / \mathrm{sfw}
$$

Where: $\mathrm{dw}=$ total dry weight $(\mathrm{kg})$; sdw $=$ dry weight of the sample $(\mathrm{g}) ; \mathrm{fw}=$ total fresh weight $(\mathrm{kg}) ; \mathrm{sfw}=$ fresh weight of the sample (g).

In the first stage of developing allometric equations for estimating AGB in the study sites, the five selected allometric equations of AGB were tested:

$$
\begin{aligned}
& y=a+b x \\
& y=a x^{b} \\
& y=a+b(\ln x) \\
& (\ln y)=a+b x \\
& (\ln y)=a+b(\ln x)
\end{aligned}
$$

Where: $y=$ total dry weight or biomass of each plant part, such as trunk, branches, leaves, and total above ground biomass (TAGB) $(\mathrm{kg}) ; \mathrm{x}=$ diameter at breast height $(\mathrm{DBH}, \mathrm{cm})$, total height $(\mathrm{H}$, meter $)$, and $\left(\mathrm{DBH}^{2} \times \mathrm{H}\right)\left(\mathrm{cm}^{2}\right.$ m); ' $a$ ' and ' $b$ ' = coefficients estimated by regression.

All regression analyses were carried out using SPSS version 18 for windows (SPSS Japan, Tokyo, Japan). The $\mathrm{R}^{2}$ values were determined to evaluate precision among all tested allometric equations. To choose the most appropriate regression, several stages, such as analyses of all tested possible regressions, elimination of the inappropriate regressions, and then selection of the best regression were carried out. The best regression was selected based on the goodness of fit with focusing on the suitable scatter plot, good $P$ values and the high values of adjusted $\mathrm{R}^{2}$ among all tested regressions. 


\section{RESULTS AND DISCUSSION}

\section{The height and diameter of selected sample trees}

The DBH and height classes of selected sample trees for estimation of AGB are shown in Figure 1. The DBH range was $5.0-17.4 \mathrm{~cm}$ and height was $5.0-12.5 \mathrm{~m}$ for selected sample trees in the 5-year-old secondary forest (Table 1). In 10-year-old secondary forest, the destructive sample trees varied from 5.9 to $32.9 \mathrm{~cm}$ in DBH and 6.0 to $21.0 \mathrm{~m}$ in height as shown in Table 2. The harvested trees ranged from 5.7 to $41.0 \mathrm{~cm}$ in DBH and from 7.0 to $22.5 \mathrm{~m}$ in height in the 20-year-old secondary forest as described in Table 3.

The DBH of sampled trees had a positive correlation with their total height (Figure 2). The equations of these relationships were " $\mathrm{H}=0.43(\mathrm{DBH})+4.95$ " $\left(\mathrm{n}=30 ; \mathrm{R}^{2}=0.55\right)$, " $\mathrm{H}=0.32(\mathrm{DBH})+7.84 " \quad\left(\mathrm{n}=30 ; \mathrm{R}^{2}=0.58\right)$, and " $\mathrm{H}=0.40$ $(\mathrm{DBH})+8.6 "\left(\mathrm{n}=30 ; \mathrm{R}^{2}=0.66\right)$ in the 5-, 10-, and 20- yearold secondary forests, respectively. The ' $\mathrm{H}$ ' is total height (m) and 'DBH' is diameter at breast height $(\mathrm{cm})$.

\section{Above-ground biomass of trees}

Tables 1, 2, and 3 summarize the DBH, total height $(\mathrm{H})$, dry weight $(\mathrm{kg})$ of tree part biomass, and TAGB in the 5-, $10-$, and 20-year old secondary forests. In the 5- year-old secondary forest, the 30 selected trees belonged to 21 genera and 14 families. The dry weight varied from 0.30 to $7.05 \mathrm{~kg}$ for leaves, 0.80 to $22.24 \mathrm{~kg}$ for branches, 1.50 to $49.12 \mathrm{~kg}$ for trunk, and 2.04 to $68.78 \mathrm{~kg}$ for TAGB in 5year-old secondary forest. Artocarpus elasticus Reinw. with $5.1 \mathrm{~cm} \mathrm{DBH}$ and $6.9 \mathrm{~m}$ height was the only sample without branch. This species had the lowest dry weight of trunk $(1.50 \mathrm{~kg})$ and TAGB $(2.04 \mathrm{~kg})$ among all selected species. Generally, the high value of DBH and total height were correlated with the high dry weight of leaf, branch, trunk, and TAGB for sample trees in this study site as mentioned in Table 1.

The 30 selected species belonged to 19 genera and 12 families in the 10-year-old secondary forest. The dry weight of trees varied from $0.50-39.68 \mathrm{~kg}, 1.06-87.96 \mathrm{~kg}$, $2.73-280.81 \mathrm{~kg}$, and $4.29-408.44 \mathrm{~kg}$ for leaves, branches, trunk, and TAGB, respectively. Several species of the genera Alstonia, Cratoxylum, Macaranga, and Litsea showed variation in terms of dry weight of plant parts. The results showed that the largest and tallest sample tree reached the highest dry weight of all plant parts, and vice versa. Most of the selected trees with higher values of DBH and total height also had higher values of dry weight of tree parts in the 10-year-old secondary forest as presented in Table 2 .

The 30 selected trees in the 20-year-old secondary forest were included in 26 genera and 21 families. The dry weight of leaves, branches, trunk, and TAGB varied from 0.16 to $34.48 \mathrm{~kg}, 2.15$ to $163.54 \mathrm{~kg}, 4.38$ to $525.90 \mathrm{~kg}$, and 7.97-683.91 kg, respectively (Table 3). The variation of dry weight in different plant parts was shown by Cratoxylum spp. and Artocarpus spp. The largest selected tree reached the highest dry weight of tree part biomass, while the smallest one had the lowest dry weight in the 20-year-old secondary forest. The higher values of dry weight of all tree parts were shown by the selected trees which had the larger DBH and total height.

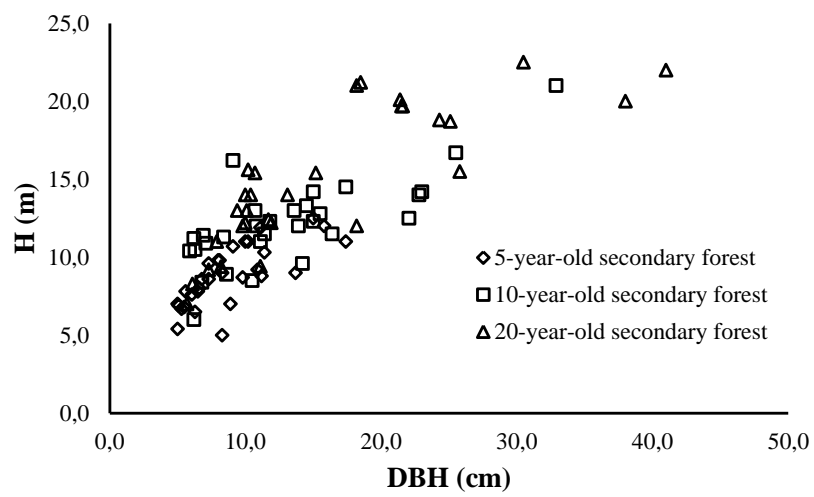

Figure 2. The DBH and total height of sampled trees to develop alometric equations. $\diamond 5$-year-old secondary forest : " $\mathrm{H}=0.43$ $(\mathrm{DBH})+4.95 "\left(\mathrm{n}=30, \mathrm{R}^{2}=0.55\right) ; \square 10$-year-old secondary forest : "H=0.32 (DBH) + 7.84" $\left(\mathrm{n}=30, \mathrm{R}^{2}=0.58\right) ; \triangle 20$-year-old secondary forest : " $\mathrm{H}=0.40(\mathrm{DBH})+8.6$ " $\left(\mathrm{n}=30, \mathrm{R}^{2}=0.66\right)$
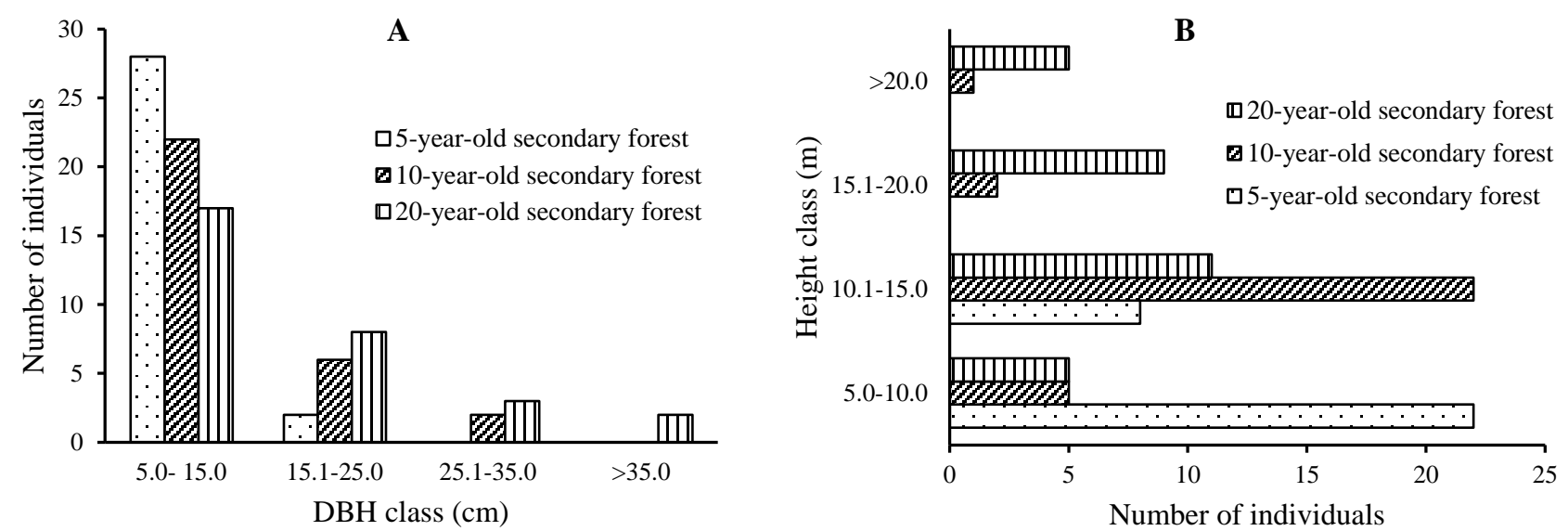

Figure 1. The distributions of (A) DBH classes and (B) height classes of sample trees to develop allometric equations. 


\section{Wood density}

The relationship between DBH and WD of the selected sample trees was illustrated in Figure 3. The equations of these relationship were "WD=-0.01 $(\mathrm{DBH})+0.52$ " $(\mathrm{n}=30$; $\left.\mathrm{R}^{2}=0.10\right)$, "WD=0.01 (DBH)+0.33" $\left(\mathrm{n}=30 ; \mathrm{R}^{2}=0.09\right)$, and "WD=-0.01 $(\mathrm{DBH})+0.52 "\left(\mathrm{n}=30 ; \mathrm{R}^{2}=0.11\right)$ in the 5,10 , and 20-year-old secondary forests, respectively. This indicated that WD did not relate to $\mathrm{DBH}$. The results showed that increasing DBH was not followed by an increase in WD density. This trend was similar to the result of previous studies by Baker et al. (2004), Basuki et al. (2009), and Nogueira et al. (2005, 2007).

The average WD of harvested trees in this study was $0.42 \mathrm{~g} \mathrm{~cm}^{-3}, 0.39 \mathrm{~g} \mathrm{~cm}^{-3}$, and $0.45 \mathrm{~g} \mathrm{~cm}^{-3}$ for the $5-10$ - and 20 year-old secondary forests as shown in Tables 1, 2, and 3 . These values were lower than those reported by Kenzo et al. (2009a), Kiyono and Hastaniah (2005), and Nelson et al. (1999). The mixed species of logged-over tropical rain forest in Sabal and Balai Ringin, Sarawak, Malaysia had average WD of $0.497 \mathrm{~g} \mathrm{~cm}^{-3}$ (Kenzo et al., 2009a), while the average WD of trees in secondary forest with mainly Schima wallichii in Kalimantan, Indonesia was $0.67 \mathrm{~g} \mathrm{~cm}^{-3}$ (Kiyono and Hastaniah 2005), and the average WD of mixed species of secondary forest in Central Amazon was $0.54 \mathrm{~g} \mathrm{~cm}^{-3}$ (Nelson et al. 1999).

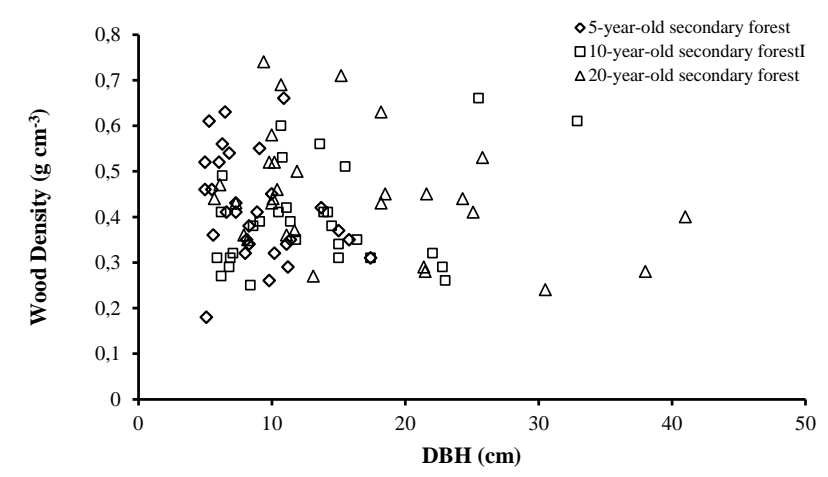

Figure 3. Relationship between DBH and wood density of selected sample trees to assessed allometric equations. $\diamond 5$-yearold secondary forest, $\mathrm{WD}=-0.01(\mathrm{DBH})+0.52\left(\mathrm{n}=30 ; \mathrm{R}^{2}=0.10\right)$; $\square$ 10-year-old secondary forest, WD $=0.01(\mathrm{DBH})+0.33(\mathrm{n}=30$; $\left.\mathrm{R}^{2}=0.09\right) ; \triangle 20$-year-old secondary forest, $\mathrm{WD}=-0.01(\mathrm{DBH})+$ $0.52\left(\mathrm{n}=30 ; \mathrm{R}^{2}=0.11\right)$

Table 1. All data sets for diameter at breast height $(\mathrm{DBH})$, total height $(\mathrm{H})$, dry weight $(\mathrm{kg})$ of tree part biomass, total above-ground biomass (TAGB) and wood density (WD, $\mathrm{g} \mathrm{cm}^{-3}$ ) in the 5-year-old secondary forest

\begin{tabular}{|c|c|c|c|c|c|c|c|c|c|}
\hline $\begin{array}{l}\text { Tree } \\
\text { Code }\end{array}$ & Family & Species & $\begin{array}{r}\text { DBH } \\
(\mathbf{c m})\end{array}$ & $\begin{array}{l}\mathbf{H} \\
(\mathbf{m})\end{array}$ & $\begin{array}{c}\text { Leaves } \\
\text { (kg) }\end{array}$ & $\begin{array}{c}\text { Branches } \\
(\mathrm{kg})\end{array}$ & $\begin{array}{c}\text { Trunk } \\
\text { (kg) }\end{array}$ & $\begin{array}{c}\text { TAGB } \\
\text { (kg) }\end{array}$ & $\begin{array}{c}\text { WD } \\
\left(\mathrm{g} \mathrm{cm}^{-3}\right)\end{array}$ \\
\hline T1 & Euphorbiaceae & Macaranga gigantea Mull. Arg. & 11.2 & 8.8 & 4.22 & 13.68 & 14.11 & 32.01 & 0.29 \\
\hline $\mathrm{T} 2$ & Rubiaceae & Nauclea subdita Merr. & 8.0 & 9.8 & 1.16 & 2.69 & 8.31 & 12.16 & 0.32 \\
\hline $\mathrm{T} 3$ & Euphorbiaceae & Endospermum diadenum (Miq.) Airy Shaw & 11.1 & 11.9 & 2.50 & 4.39 & 20.09 & 26.98 & 0.34 \\
\hline $\mathrm{T} 4$ & Clusiaceae & Cratoxylum arborescens Blume. & 11.4 & 10.3 & 5.47 & 8.05 & 18.61 & 32.14 & 0.35 \\
\hline T5 & Dilleniaceae & Dillenia suffruticosa Martelli & 8.3 & 5.0 & 0.91 & 2.91 & 4.03 & 7.85 & 0.38 \\
\hline T6 & Euphorbiaceae & Macaranga trichocarpa Mull. Arg. & 8.1 & 9.8 & 1.71 & 3.27 & 7.71 & 12.69 & 0.35 \\
\hline $\mathrm{T} 7$ & Rhamnaceae & Alphitonia excelsa Reissek ex Endl. & 10.0 & 11.0 & 2.18 & 4.70 & 16.54 & 23.42 & 0.45 \\
\hline $\mathrm{T} 8$ & Myrtaceae & Syzygium polyanthum Walp. & 10.9 & 9.2 & 6.65 & 22.24 & 22.80 & 51.68 & 0.66 \\
\hline T9 & Verbenaceae & Callicarpa longifolia Lam. & 8.3 & 9.0 & 0.79 & 6.34 & 19.41 & 26.55 & 0.34 \\
\hline $\mathrm{T} 10$ & Verbenaceae & Vitex pubescens Vahl. & 9.1 & 10.7 & 2.01 & 7.77 & 20.43 & 30.20 & 0.55 \\
\hline $\mathrm{T} 11$ & Euphorbiaceae & Glochidion arborescens Blume. & 7.3 & 9.6 & 2.51 & 3.04 & 8.67 & 14.21 & 0.43 \\
\hline $\mathrm{T} 12$ & Rubiaceae & Timonius flavescens Baker & 6.1 & 7.6 & 2.23 & 4.33 & 8.84 & 15.40 & 0.52 \\
\hline $\mathrm{T} 13$ & Apocynaces & Alstonia scholaris (L.) R. Br. & 9.8 & 8.7 & 1.53 & 1.67 & 8.03 & 11.22 & 0.26 \\
\hline $\mathrm{T} 14$ & Moraceae & Ficus & 5.0 & 5.4 & 0.44 & 2.46 & 3.85 & 6.75 & 0.52 \\
\hline $\mathrm{T} 15$ & Euphorbiaceae & riloba Mull. Arg. & 10.2 & 11.0 & 3.07 & 3.82 & 15.94 & 22.84 & 0.32 \\
\hline T16 & Theaceae & Adinandra dumosa Jack & 13.7 & 9.0 & 7.05 & 4.36 & 18.62 & 30.03 & 0.42 \\
\hline $\mathrm{T} 17$ & Euphorbiaceae & Mallotus macrostachyus Mull. Arg. & 6.6 & 8.0 & 0.57 & 2.22 & 6.45 & 9.24 & 0.41 \\
\hline $\mathrm{T} 18$ & Moraceae & Artocarpus elasticus Reinw. & 5.1 & 6.9 & 0.54 & - & 1.50 & 2.04 & 0.18 \\
\hline T19 & Theaceae & Ploiarium alternifolium Melchior. & 6.5 & 7.8 & 0.32 & 2.32 & 5.99 & 8.63 & 0.63 \\
\hline $\mathrm{T} 20$ & Loganiaceae & Fagraea resinosa Leenh. & 6.8 & 8.6 & 0.88 & 6.05 & 7.90 & 14.82 & 0.54 \\
\hline $\mathrm{T} 21$ & Euphorbiaceae & Macaranga hypoleuca Mull. Arg. & 17.4 & 11.0 & 5.92 & 14.81 & 31.34 & 52.06 & 0.31 \\
\hline $\mathrm{T} 22$ & Aquifoliaceae & Ilex cymosa Blume & 5.0 & 7.0 & 0.87 & 0.80 & 3.35 & 5.02 & 0.46 \\
\hline $\mathrm{T} 23$ & Moraceae & Ficus condensa King. & 5.5 & 6.8 & 0.39 & 2.27 & 3.42 & 6.08 & 0.46 \\
\hline $\mathrm{T} 24$ & Euphorbiaceae & Macaranga beccariana Merr. & 5.6 & 7.8 & 0.41 & 0.96 & 2.73 & 4.10 & 0.36 \\
\hline $\mathrm{T} 25$ & Clusiaceae & Cratoxylum formosum Benth. \& Hook. f. ex Dyer & 5.3 & 6.7 & 0.30 & 1.41 & 4.96 & 6.66 & 0.61 \\
\hline $\mathrm{T} 26$ & Clusiaceae & Cratoxylum glaucum Korth. & 6.3 & 6.5 & 1.14 & 1.72 & 4.04 & 6.90 & 0.56 \\
\hline $\mathrm{T} 27$ & Asteraceae & Vernonia arborea Buch. Ham. & 7.3 & 8.6 & 2.23 & 2.48 & 6.96 & 11.68 & 0.41 \\
\hline $\mathrm{T} 28$ & Moraceae & Artocarpus dadak Miq. & 8.9 & 7.0 & 0.59 & 1.12 & 3.44 & 5.14 & 0.41 \\
\hline $\mathrm{T} 29$ & Apocynaceae & Alstonia scholaris (L.) R. Br. & 15.8 & 12.0 & 2.09 & 13.37 & 39.95 & 55.41 & 0.35 \\
\hline $\mathrm{T} 30$ & Rubiaceae & Euodia glabra (B1.) B1. & 15.0 & 12.5 & 4.12 & 15.54 & 49.12 & 68.78 & 0.37 \\
\hline \multicolumn{3}{|c|}{ Total } & 265.5 & 264.0 & 64.78 & 160.78 & 387.13 & 612.69 & 12.56 \\
\hline \multicolumn{3}{|c|}{ Average } & 8.9 & 8.8 & 2.16 & 5.54 & 12.90 & 20.42 & 0.42 \\
\hline \multicolumn{3}{|c|}{ Minimum } & 5.0 & 5.0 & 0.30 & 0.80 & 1.50 & 2.04 & 0.18 \\
\hline \multicolumn{3}{|c|}{ Maximum } & 17.4 & 12.5 & 7.05 & 22.24 & 49.12 & 68.78 & 0.66 \\
\hline
\end{tabular}

Note: $\mathrm{DBH}=$ diameter at breast height; $\mathrm{H}=$ height; TAGB = total above ground biomass; $\mathrm{WD}=$ wood density. 
Table 2. All data sets for diameter at breast height $(\mathrm{DBH})$, total height $(\mathrm{H})$, dry weight $(\mathrm{kg})$ of tree part biomass, total above-ground biomass (TAGB), and wood density (WD, $\mathrm{g} \mathrm{cm}^{-3}$ ) in the 10 -year-old secondary forest

\begin{tabular}{|c|c|c|c|c|c|c|c|c|c|}
\hline $\begin{array}{l}\text { Tree } \\
\text { code }\end{array}$ & Family & Species & $\begin{array}{r}\text { DBH } \\
(\mathbf{c m})\end{array}$ & $\begin{array}{l}\mathbf{H} \\
(\mathbf{m})\end{array}$ & $\begin{array}{c}\begin{array}{c}\text { Leaves } \\
(\mathrm{kg})\end{array} \\
\end{array}$ & $\begin{array}{c}\text { Branches } \\
(\mathrm{kg})\end{array}$ & $\begin{array}{c}\text { Trunk } \\
\text { (kg) }\end{array}$ & $\begin{array}{c}\text { TAGB } \\
(\mathbf{k g})\end{array}$ & $\begin{array}{c}\text { WD } \\
\left(\mathrm{g} \mathrm{cm}^{-3}\right)\end{array}$ \\
\hline$\overline{\mathrm{P} 1}$ & Clusiaceae & Cratoxylum arborescens Blume. & 15.0 & 12.3 & 9.26 & 16.31 & 41.09 & 66.65 & 0.34 \\
\hline $\mathrm{P} 2$ & Theaceae & Adinandra dumosa Jack & 13.9 & 12.0 & 10.74 & 10.41 & 42.04 & 63.19 & 0.41 \\
\hline P3 & Dilleniaceae & Dillenia suffruticosa Martelli & 8.6 & 8.9 & 0.65 & 6.36 & 8.63 & 15.64 & 0.38 \\
\hline P4 & Apocynaceae & Alstonia pneumatophora Backer ex Den Berger & 13.6 & 13.0 & 5.87 & 16.22 & 65.84 & 87.93 & 0.56 \\
\hline P5 & Euphorbiaceae & Macaranga triloba Mull. Arg. & 14.5 & 13.3 & 2.42 & 11.65 & 44.84 & 58.91 & 0.38 \\
\hline P6 & Moraceae & Ficus aurata Miq. & 10.5 & 8.5 & 1.78 & 10.77 & 12.66 & 25.22 & 0.41 \\
\hline P7 & Asteraceae & Vernonia arborea Buch. Ham. & 17.4 & 14.5 & 3.09 & 9.74 & 58.57 & 71.39 & 0.31 \\
\hline P8 & Euphorbiaceae & Macaranga hypoleuca Mull. Arg. & 11.4 & 11.5 & 1.88 & 6.35 & 22.39 & 30.61 & 0.39 \\
\hline P9 & Verbenaceae & Clerodendron sp. & 10.7 & 13.0 & 4.33 & 8.96 & 30.58 & 43.86 & 0.60 \\
\hline $\mathrm{P} 10$ & Euphorbiaceae & Glochidion arborescens & 10.8 & 12.0 & 1.15 & 7.31 & 21.65 & 30.11 & 0.53 \\
\hline $\mathrm{P} 11$ & Euphorbiaceae & Macaranga gigantea Mull. Arg. & 22.8 & 14.0 & 17.29 & 53.60 & 75.93 & 146.82 & 0.29 \\
\hline $\mathrm{P} 12$ & Moraceae & us elasticus Reinw. & 23.0 & 14.2 & 4.42 & 17.84 & 68.39 & 90.65 & 0.26 \\
\hline $\mathrm{P} 13$ & Rutaceae & Euodia glabra (B1.) Bl. & 16.4 & 11.5 & 1.81 & 7.42 & 49.87 & 59.10 & 0.35 \\
\hline $\mathrm{P} 14$ & Clusiaceae & Cratoxylum formosum Benth. \& & 11.1 & 11.0 & 1.18 & 6.85 & 15.03 & 23.06 & 0.42 \\
\hline $\mathrm{P} 15$ & Euphorbiaceae & Endospermum diadenum (Miq.) Airy Shaw & 14.2 & 9.6 & 1.07 & 5.59 & 22.11 & 28.78 & 0.41 \\
\hline P16 & Dipterocarpaceae & Shorea macrophylla (de Vriese) P.S. Ashton & 15.0 & 14.2 & 2.26 & 4.77 & 29.61 & 36.64 & 0.31 \\
\hline P17 & Euphorbiaceae & Macaranga pruinosa Mull. Arg. & 22.1 & 12.5 & 35.59 & 41.19 & 67.66 & 144.44 & 0.32 \\
\hline $\mathrm{P} 18$ & Euphorbiaceae & Macaranga caladifolia Becc. & 7.1 & 10.9 & 0.74 & 3.21 & 5.26 & 9.20 & 0.32 \\
\hline P19 & Asteraceae & Ilex cymosa Blume & 11.8 & 12.3 & 2.50 & 3.46 & 18.65 & 24.61 & 0.35 \\
\hline $\mathrm{P} 20$ & Euphorbiaceae & Aporosa sp. & 5.9 & 10.4 & 0.55 & 1.59 & 4.97 & 7.11 & 0.31 \\
\hline $\mathrm{P} 21$ & Lauraceae & Litsea crassifolia Boer & 6.9 & 11.4 & 0.86 & 1.99 & 7.72 & 10.56 & 0.31 \\
\hline $\mathrm{P} 22$ & Euphorbiaceae & Macaranga beccariana Merr. & 8.4 & 11.3 & 1.06 & 2.81 & 7.57 & 11.44 & 0.25 \\
\hline $\mathrm{P} 23$ & Apocynaceae & Alstonia spatulata Blume & 32.9 & 21.0 & 39.68 & 87.96 & 280.81 & 408.44 & 0.61 \\
\hline P24 & Euphorbiaceae & Macaranga lowii King ex Hook. f. & 6.8 & 8.4 & 0.73 & 1.65 & 4.65 & 7.03 & 0.29 \\
\hline $\mathrm{P} 25$ & Euphorbiaceae & Mallotus macrostachyus Mull. Arg. & 9.1 & 16.2 & 0.50 & 8.08 & 17.18 & 25.76 & 0.39 \\
\hline P26 & Lauraceae & Litsea $\mathrm{sp}$. & 6.2 & 11.2 & 0.55 & 1.92 & 6.22 & 8.69 & 0.41 \\
\hline P27 & Verbenaceae & Vitex pubescens Vahl. & 25.5 & 16.7 & 9.30 & 53.43 & 208.58 & 271.31 & 0.66 \\
\hline P28 & Apocynaceae & Alstonia scholaris (L.) R. Br. & 6.2 & 6.0 & 0.50 & 1.06 & 2.73 & 4.29 & 0.27 \\
\hline P29 & Clusiaceae & Cratoxylum glaucum Korth. & 15.5 & 12.8 & 9.36 & 25.27 & 55.77 & 90.40 & 0.51 \\
\hline P30 & Sapindaceae & Nephelium cuspidatum Blume & 6.3 & 10.5 & 1.71 & 2.89 & 12.27 & 16.88 & 0.49 \\
\hline \multicolumn{3}{|c|}{ Total } & 399.6 & 365.1 & 172.84 & 436.65 & 1309.22 & 1918.70 & 11.84 \\
\hline \multicolumn{3}{|c|}{ Average } & 13.3 & 12.2 & 5.76 & 14.55 & 43.64 & 63.96 & 0.39 \\
\hline \multicolumn{3}{|c|}{ Minimum } & 5.9 & 6.0 & 0.50 & 1.06 & 2.73 & 4.29 & 0.25 \\
\hline \multicolumn{3}{|c|}{ Maximum } & 32.9 & 21.0 & 39.68 & 87.96 & 280.81 & 408.44 & 0.66 \\
\hline
\end{tabular}

Note: $\mathrm{DBH}=$ diameter at breast height; $\mathrm{H}=$ height; TAGB = total above ground biomass; WD = wood density

The average WD of the sample trees in these study sites was higher than that of Gmelina arborea and Paraserianthes falcataria in plantation forest i.e. 0.34 and $0.32 \mathrm{~g} \mathrm{~cm}^{-3}$, respectively (Kawahara et al. 1981) and of mixed species of secondary forest in Niah and Sungai Liku, Sarawak, Malaysia, i.e., $0.35 \mathrm{~g} \mathrm{~cm}^{-3}$ (Kenzo et al. 2009a). The range of values of WD ( 0.39 to $\left.0.45 \mathrm{~g} \mathrm{~cm}^{-3}\right)$ resulted in this study was within the range of WD values reported by other studies, such as that of mixed species of moist tropical forest, i.e. $0.40-0.79 \mathrm{~g} \mathrm{~cm}^{-3}$ (Brown 1997), that of mixed species of secondary forest, i.e. $0.29-0.47 \mathrm{~g} \mathrm{~cm}^{-3}$ in East Kalimantan, Indonesia (Hashimoto et al. 2004) and in Sumatra, Indonesia, i.e. and $0.35-0.91 \mathrm{~g} \mathrm{~cm}^{-3}$ (Ketterings et al. 2001).

\section{The allometric equations for trees in the secondary forests}

The summary of the selected equations for predicting plant part biomass of subject trees in the study sites is presented in Table 4. The testing of log-linear model (ln $\mathrm{y}=\mathrm{a}+\mathrm{b} \ln \mathrm{x})$ and exponential model $\left(\mathrm{y}=\mathrm{ax} \mathrm{x}^{\mathrm{b}}\right)$ showed the good fitting to related plant parameters $\left(\mathrm{DBH},\left(\mathrm{DBH}^{2} \times \mathrm{H}\right)\right.$, or $\mathrm{H})$ and plant part biomass. For several tested relationships, the simple linear model $(\mathrm{y}=\mathrm{a}+\mathrm{bx})$ and semilog model (ln $\mathrm{y}=\mathrm{a}+\mathrm{b} \mathrm{x}$ ) had good $P$ values and high $\mathrm{R}^{2}$ values, but the scatter plots of these relationships were not the most suitable. The testing of semilog model $(y=$ $a+b \ln x)$ showed no goodness of fit for all tested parameters in terms of scatter plot and $\mathrm{R}^{2}$ values. Generally, the analyses of all tested regression in the 5-, $10-$, and 20 years old secondary forests showed many tested allometric equations having relatively high $\mathrm{R}^{2}$ values.

The $\log$ model $(\ln \mathrm{y}=\mathrm{a}+\mathrm{b} \ln \mathrm{x})$ showed that the dependent variables (leaf, branch, trunk, and AGB) of trees were highly correlated with the independent variables $\left(\mathrm{DBH}, \quad\left(\mathrm{DBH}^{2} \times \mathrm{H}\right)\right)$ in the 5-,10-, and 20-year-old secondary forests. On the other hand, the exponential model $\left(y=a x^{b}\right)$ was the good equation to relate dependent variables (leaf, branch, trunk, and AGB) of tree and tree height. The weak correlations between branch and all independent variables had relatively low $R^{2}$ values in the 5year-old secondary forest $\left(\mathrm{R}^{2}=0.38-0.53\right)$. In addition, height was as a good predictor for trunk dry biomass (in the $5-, 10-$, and 20-year-old secondary forests) and TAGB (in the 5-and 20-year-old secondary forests). 
Table 3. All data sets for diameter at breast height $(\mathrm{DBH})$, total height $(\mathrm{H})$, dry weight $(\mathrm{kg})$ of tree part biomass, total above-ground biomass (TAGB), and wood density (WD, $\mathrm{g} \mathrm{cm}^{-3}$ ) in the 20 -year-old secondary forest

\begin{tabular}{|c|c|c|c|c|c|c|c|c|c|}
\hline $\begin{array}{l}\text { Tree } \\
\text { code }\end{array}$ & Family & Species & $\begin{array}{r}\text { DBH } \\
(\mathbf{c m})\end{array}$ & $\begin{array}{l}\mathbf{H} \\
(\mathbf{m})\end{array}$ & $\begin{array}{c}\text { Leaves } \\
\text { (kg) }\end{array}$ & $\begin{array}{c}\text { Branches } \\
(\mathrm{kg})\end{array}$ & $\begin{array}{c}\text { Trunk } \\
(\mathbf{k g})\end{array}$ & $\begin{array}{c}\text { TAGB } \\
(\mathbf{k g})\end{array}$ & $\begin{array}{c}\text { WD } \\
\left(\mathrm{g} \mathrm{cm}^{-3}\right)\end{array}$ \\
\hline V1 & Euphorbiaceae & Endospermum diadenum (Miq.) Airy Shaw & 25.1 & 18.7 & 12.19 & 91.03 & 198.94 & 302.16 & 0.41 \\
\hline V2 & Theaceae & Adinandra dumosa Jack & 24.3 & 18.8 & 13.07 & 61.75 & 226.85 & 301.67 & 0.44 \\
\hline V3 & Symplocaceae & Symplocos sp. & 10.1 & 13.0 & 1.64 & 4.84 & 22.94 & 29.42 & 0.44 \\
\hline V4 & Euphorbiaceae & Glochidion arborescens Blume. & 11.9 & 12.2 & 6.60 & 15.79 & 39.32 & 61.71 & 0.50 \\
\hline V5 & Rhizophoraceae & Carallia sp. & 10.4 & 14.0 & 1.77 & 6.24 & 29.43 & 37.44 & 0.46 \\
\hline V6 & Rutaceae & Euodia glabra (Bl.) Bl. & 7.9 & 11.0 & 0.33 & 2.15 & 10.53 & 13.01 & 0.36 \\
\hline V7 & Rubiaceae & Timonius flavescens Baker & 10.7 & 15.4 & 0.23 & 4.47 & 36.58 & 41.28 & 0.69 \\
\hline V8 & Dilleniaceae & Dillenia suffruticosa Martelli & 11.1 & 9.4 & 1.24 & 20.89 & 11.23 & 33.36 & 0.36 \\
\hline V9 & Verbenaceae & Vitex pubescens Vahl. & 6.1 & 8.3 & 0.16 & 2.52 & 6.56 & 9.24 & 0.47 \\
\hline V10 & Ulmaceae & Gironniera nervosa Planch. & 10.0 & 12.2 & 6.54 & 7.39 & 23.60 & 37.54 & 0.43 \\
\hline V11 & Moraceae & Artocarpus integer (Thunb.) Merr. & 5.7 & 7.0 & 1.40 & 2.19 & 4.38 & 7.97 & 0.44 \\
\hline V12 & Sapotaceae & Palaquium decurrens H.J. Lam & 8.2 & 9.4 & 1.56 & 4.05 & 7.79 & 13.41 & 0.35 \\
\hline V13 & Fagaceae & Castanopsis sp. & 7.3 & 9.2 & 2.18 & 3.67 & 7.42 & 13.27 & 0.43 \\
\hline V14 & Rubiaceae & Nauc & 15.2 & 15.4 & 5.96 & 13.60 & 80.78 & 100.34 & 0.71 \\
\hline V15 & Moraceae & nisophyllus Miq. & 18.5 & 21.2 & 5.05 & 15.74 & 123.89 & 144.68 & 0.45 \\
\hline V16 & Myrta & polyanthum Walp. & 18.2 & 21.0 & 10.73 & 28.09 & 169.16 & 207.98 & 0.63 \\
\hline V17 & Dipterocarpaceae & Hop & 9.4 & 13.0 & 6.18 & 21.86 & 39.78 & 67.81 & 0.74 \\
\hline V18 & Moraceae & us dadak Miq. & 9.8 & 12.0 & 1.33 & 9.60 & 14.52 & 25.45 & 0.52 \\
\hline V19 & Lauraceae & elliptica Blume & 11.7 & 12.4 & 2.95 & 5.27 & 22.75 & 30.98 & 0.37 \\
\hline V20 & Anacardiaceae & Campnosperma auriculatum (Blume) Hook. f. & 21.5 & 19.7 & 6.67 & 19.59 & 107.42 & 133.68 & 0.28 \\
\hline $\mathrm{V} 21$ & Sapindaceae & idatum Blume & 10.0 & 14.0 & 4.10 & 5.41 & 43.43 & 52.95 & 0.58 \\
\hline $\mathrm{V} 22$ & Burseraceae & (Blume) H.J. Lam & 10.2 & 15.6 & 0.94 & 5.63 & 19.75 & 26.32 & 0.52 \\
\hline $\mathrm{V} 23$ & Clusiaceae & $m$ Benth. \& Hook. f. ex Dyer & 25.8 & 15.5 & 22.61 & 68.00 & 197.68 & 288.28 & 0.53 \\
\hline V24 & Loganiaceae & Norrisia malaccensis Gardn. & 18.2 & 12.0 & 2.63 & 19.92 & 52.96 & 75.51 & 0.43 \\
\hline V25 & Clusiaceae & Cratoxylum arborescens Blume & 41.0 & 22.0 & 34.48 & 123.53 & 525.90 & 683.91 & 0.40 \\
\hline V26 & Euphorbiaceae & Aporosa sp. & 30.5 & 22.5 & 23.12 & 112.84 & 387.73 & 523.69 & 0.24 \\
\hline $\mathrm{V} 27$ & Clusiaceae & Cratoxylum glaucum Korth. & 38.0 & 20.0 & 22.57 & 163.54 & 466.48 & 652.58 & 0.28 \\
\hline V28 & Rhizophoraceae & Pellacalyx axillaris Korth. & 13.1 & 14.0 & 1.39 & 7.69 & 43.30 & 52.37 & 0.27 \\
\hline V29 & Rutaceae & Timonius borneensis Valeton & 21.4 & 20.1 & 7.31 & 9.78 & 147.75 & 164.84 & 0.29 \\
\hline V30 & Apocynaceae & Alstonia spatulata Blume & 21.6 & 19.7 & 8.08 & 16.28 & 185.40 & 209.76 & 0.45 \\
\hline \multicolumn{3}{|c|}{ Total } & 482.9 & 448.7 & 215.02 & 873.36 & 3254.23 & 4342.62 & 13.47 \\
\hline \multicolumn{3}{|c|}{ Average } & 16.1 & 15.0 & 7.17 & 29.11 & 108.47 & 144.75 & 0.45 \\
\hline \multicolumn{3}{|c|}{ Minimum } & 5.7 & 7.0 & 0.16 & 2.15 & 4.38 & 7.97 & 0.24 \\
\hline \multicolumn{3}{|c|}{ Maximum } & 41.0 & 22.5 & 34.48 & 163.54 & 525.90 & 683.91 & 0.74 \\
\hline
\end{tabular}

Note: $\mathrm{DBH}=$ diameter at breast height; $\mathrm{H}=$ height; TAGB = total above ground biomass; WD = wood density

The allometric equation of "In $(\mathrm{AGB})=\mathrm{a}+\mathrm{b} \ln (\mathrm{DBH})$ " to estimate the AGB of different forest types was also reported by Basuki et al. (2009), Brown (1997), Chamber et al. (2001), Hashimoto et al. (2004), Kawahara et al. (1981), Nelson et al. (1999), Rai and Proctor (1986), Sierra et al. (2007) and Yamakura et al. (1986). In contrast, Kenzo et al. (2009a), Kenzo et al. (2009b), and Kiyono and Hastaniah (2005) proposed the model of "In $(A G B)=a \times$ $(\mathrm{DBH})$ " " to estimate AGB. A study on distribution of AGB of Endospermum diadenum (Miq.) Airy Shaw (Terbulan) and its relationship with $\mathrm{DBH}$ and age in Sabal Forest Reserve, Sri Aman showed that foliage branch, stem, and total above ground was highly correlated both with DBH $\left(r^{2} \geq 0.90\right)$ and with age $\left(r^{2} \geq 0.86\right)$ (Bohari, 2007). The developed allometric equations to estimate total AGB as a function of $\mathrm{DBH}$ and $\mathrm{H}$ showed strong correlation with $\mathrm{R}^{2}$ of 0.97 to 0.99 . There was also a relatively strong correlation for allometric relationship between $\left(\mathrm{DBH}^{2} \times \mathrm{H}\right)$ and AGB in the logged-over tropical rainforests in Sarawak, Malaysia (Kenzo et al. 2009a).

\section{Comparison among various allometric equations}

The estimates of AGB using previously reported relationships for trees with $\mathrm{DBH}$ of $\geq 5 \mathrm{~cm}$ at secondary forests of different ages in the study sites are presented in Table 5. Figure 4 illustrates the comparison with previously reported relationship between AGB and DBH in the study sites. The previous studies of allometric equations for various types of tropical forests were chosen to give comparison on estimated AGB in the study sites. The estimated $\mathrm{AGB}$ was $10.17 \mathrm{Mg} \mathrm{ha}^{-1}$ in the 5-year-old secondary forest, higher than other estimates using equations by Hashimoto et al. (2004), i.e., $9.80 \mathrm{Mg} \mathrm{ha}^{-1}$ and Kenzo et al. (2009b), i.e., $9.79 \mathrm{Mg} \mathrm{ha}^{-1}$. A similar value of AGB (10.50 Mg ha-1) was obtained using equation by Kettering et al. (2001). The value resulted by the developed allometric equation was lower than those using other previous reported equations, i.e., $40.61 \mathrm{Mg} \mathrm{ha}^{-1}$ (Rai and Proctor 1986)), 17.52 $\mathrm{Mg} \mathrm{ha}^{-1}$ (Yamakura et. al. 1986), 17.24 $\mathrm{Mg} \mathrm{ha}^{-1}$ (Brown 1997), $15.48 \mathrm{Mg} \mathrm{ha}^{-1}$ (Nelson et al. 1999), $20.26 \mathrm{Mg} \mathrm{ha}^{-1}$ (Chamber et. al. 2001), $14.52 \mathrm{Mg} \mathrm{ha}^{-1}$ (Kiyono and Hastaniah 2008), $12.47 \mathrm{Mg} \mathrm{ha}^{-1}$ (Sierra et. al. 2007), 22.03 $\mathrm{Mg} \mathrm{ha}^{-1}$ (Basuki et. al. 2009), and $14.98 \mathrm{Mg}$ ha $^{-1}$ (Kenzo et al. 2009a) (Table 5 and Figure 4.A). 
Table 4. The best selected allometric equations for predicting plant part biomass of trees $(\mathrm{DBH}$ of $\geq 5 \mathrm{~cm})$ in the study sites

\begin{tabular}{|c|c|c|c|c|}
\hline Dependent variable (y) & Independent variable $(x)$ & Equation & $P$ value & Adjusted $\mathbf{R}^{2}$ \\
\hline \multicolumn{5}{|l|}{ 5-year-old secondary forest } \\
\hline \multirow[t]{3}{*}{ Leaf dry biomass (kg) } & $\mathrm{DBH}(\mathrm{cm})$ & $\ln (y)=2.0576 \times \ln (x)-3.99$ & $<0.001$ & 0.60 \\
\hline & $\left(\mathrm{DBH}^{2} \times \mathrm{H}\right)\left(\mathrm{cm}^{2} \mathrm{~m}\right)$ & $\ln (y)=0.8590 \times \ln (x)-5.12$ & $<0.001$ & 0.63 \\
\hline & $\mathrm{H}(\mathrm{m})$ & $\mathrm{y}=0.08(\mathrm{x})^{0.3241}$ & $<0.001$ & 0.43 \\
\hline \multirow[t]{3}{*}{ Branch dry biomass (kg) } & $\mathrm{DBH}(\mathrm{cm})$ & $\ln (y)=1.8312 \times \ln (x)-2.57$ & $<0.001$ & 0.53 \\
\hline & $\left(\mathrm{DBH}^{2} \times \mathrm{H}\right)\left(\mathrm{cm}^{2} \mathrm{~m}\right)$ & $\ln (y)=0.7505 \times \ln (x)-3.47$ & $<0.001$ & 0.55 \\
\hline & $\mathrm{H}(\mathrm{m})$ & $\mathrm{y}=0.30(\mathrm{x})^{0.2845}$ & $<0.001$ & 0.38 \\
\hline \multirow[t]{3}{*}{ Stem dry biomass $(\mathrm{kg})$} & $\mathrm{DBH}(\mathrm{cm})$ & $\ln (y)=2.0738 \times \ln (x)-2.18$ & $<0.001$ & 0.74 \\
\hline & $\left(\mathrm{DBH}^{2} \times \mathrm{H}\right)\left(\mathrm{cm}^{2} \mathrm{~m}\right)$ & $\ln (y)=0.8759 \times \ln (x)-3.38$ & $<0.001$ & 0.81 \\
\hline & $\mathrm{H}(\mathrm{m})$ & $\mathrm{y}=0.33(\mathrm{x})^{0.3773}$ & $<0.001$ & 0.72 \\
\hline \multirow[t]{3}{*}{ Above ground biomass (kg) } & $\mathrm{DBH}(\mathrm{cm})$ & $\ln (y)=2.0859 \times \ln (x)-1.75$ & $<0.001$ & 0.73 \\
\hline & $\left(\mathrm{DBH}^{2} \times \mathrm{H}\right)\left(\mathrm{cm}^{2} \mathrm{~m}\right)$ & $\ln (y)=0.8703 \times \ln (x)-2.88$ & $<0.001$ & 0.78 \\
\hline & $\mathrm{H}(\mathrm{m})$ & $\mathrm{y}=0.62(\mathrm{x})^{0.3569}$ & $<0.001$ & 0.62 \\
\hline \multicolumn{5}{|l|}{ 10-year-old secondary forest } \\
\hline \multirow[t]{3}{*}{ Leaf dry biomass $(\mathrm{kg})$} & $\mathrm{DBH}(\mathrm{cm})$ & $\mathrm{y}=0.29(\mathrm{x})^{0.1608}$ & $<0.001$ & 0.70 \\
\hline & $\left(\mathrm{DBH}^{2} \times \mathrm{H}\right)\left(\mathrm{cm}^{2} \mathrm{~m}\right)$ & $\ln (y)=0.9466 \times \ln (x)-6.14$ & $<0.001$ & 0.69 \\
\hline & $\mathrm{H}(\mathrm{m})$ & $\mathrm{y}=0.09(\mathrm{x})^{0.2752}$ & $<0.001$ & 0.36 \\
\hline \multirow{3}{*}{ Branch dry biomass (kg) } & $\mathrm{DBH}(\mathrm{cm})$ & $\ln (y)=2.1627 \times \ln (x)-3.32$ & $<0.001$ & 0.81 \\
\hline & $\left(\mathrm{DBH}^{2} \times \mathrm{H}\right)\left(\mathrm{cm}^{2} \mathrm{~m}\right)$ & $\ln (y)=0.9178 \times \ln (x)-4.77$ & $<0.001$ & 0.82 \\
\hline & $\mathrm{H}(\mathrm{m})$ & $y=5.0354(x)-46.73$ & $<0.001$ & 0.50 \\
\hline \multirow[t]{3}{*}{ Stem dry biomass (kg) } & $\mathrm{DBH}(\mathrm{cm})$ & $\ln (y)=2.2849 \times \ln (x)-2.51$ & $<0.001$ & 0.88 \\
\hline & $\left(\mathrm{DBH}^{2} \times \mathrm{H}\right)\left(\mathrm{cm}^{2} \mathrm{~m}\right)$ & $\ln (y)=0.9882 \times \ln (x)-4.18$ & $<0.001$ & 0.93 \\
\hline & $\mathrm{H}(\mathrm{m})$ & $\mathrm{y}=0.47(\mathrm{x})^{0.3220}$ & $<0.001$ & 0.63 \\
\hline \multirow{3}{*}{ Above ground biomass (kg) } & $\mathrm{DBH}(\mathrm{cm})$ & $\ln (y)=2.2725 \times \ln (x)-2.09$ & $<0.001$ & 0.90 \\
\hline & $\left(\mathrm{DBH}^{2} \times \mathrm{H}\right)\left(\mathrm{cm}^{2} \mathrm{~m}\right)$ & $\ln (y)=0.9751 \times \ln (x)-3.70$ & $<0.001$ & 0.93 \\
\hline & $\mathrm{H}(\mathrm{m})$ & $\mathrm{y}=0.80(\mathrm{x})^{0.3092}$ & $<0.001$ & 0.59 \\
\hline \multicolumn{5}{|l|}{ 20-year-old secondary forest } \\
\hline \multirow[t]{3}{*}{ Leaf dry biomass $(\mathrm{kg})$} & $\mathrm{DBH}(\mathrm{cm})$ & $\ln (y)=2.0583 \times \ln (x)-4.17$ & $<0.001$ & 0.63 \\
\hline & $\left(\mathrm{DBH}^{2} \times \mathrm{H}\right)\left(\mathrm{cm}^{2} \mathrm{~m}\right)$ & $\ln (y)=0.8075 \times \ln (x)-5.14$ & $<0.001$ & 0.62 \\
\hline & $\mathrm{H}(\mathrm{m})$ & $\mathrm{y}=0.15(\mathrm{x})^{0.2101}$ & $<0.001$ & 0.46 \\
\hline \multirow[t]{3}{*}{ Branch dry biomass (kg) } & $\mathrm{DBH}(\mathrm{cm})$ & $\mathrm{y}=1.89(\mathrm{x})^{0.1211}$ & $<0.001$ & 0.80 \\
\hline & $\left(\mathrm{DBH}^{2} \times \mathrm{H}\right)\left(\mathrm{cm}^{2} \mathrm{~m}\right)$ & $\ln (y)=0.8140 \times \ln (x)-3.87$ & $<0.001$ & 0.77 \\
\hline & $\mathrm{H}(\mathrm{m})$ & $\mathrm{y}=0.68(x)^{0.1991}$ & $<0.001$ & 0.50 \\
\hline \multirow[t]{3}{*}{ Stem dry biomass (kg) } & $\mathrm{DBH}(\mathrm{cm})$ & $\ln (y)=2.4558 \times \ln (x)-2.59$ & $<0.001$ & 0.92 \\
\hline & $\left(\mathrm{DBH}^{2} \times \mathrm{H}\right)\left(\mathrm{cm}^{2} \mathrm{~m}\right)$ & $\ln (\mathrm{y})=0.9898 \times \ln (\mathrm{x})-3.96$ & $<0.001$ & 0.95 \\
\hline & $\mathrm{H}(\mathrm{m})$ & $\mathrm{y}=0.72(\mathrm{x})^{0.2820}$ & $<0.001$ & 0.85 \\
\hline \multirow[t]{3}{*}{ Above ground biomass (kg) } & $\mathrm{DBH}(\mathrm{cm})$ & $\ln (y)=2.3207 \times \ln (x)-1.89$ & $<0.001$ & 0.93 \\
\hline & $\left(\mathrm{DBH}^{2} \times \mathrm{H}\right)\left(\mathrm{cm}^{2} \mathrm{~m}\right)$ & $\ln (\mathrm{y})=0.9277 \times \ln (\mathrm{x})-3.12$ & $<0.001$ & 0.95 \\
\hline & $\mathrm{H}(\mathrm{m})$ & $\mathrm{y}=1.50(\mathrm{x})^{0.2559}$ & $<0.001$ & 0.80 \\
\hline
\end{tabular}

Note: $P$ values of the regression analysis are shown. Adjusted $\mathrm{R}^{2}$ denotes multiple coefficients of determination

In the 10-year-old secondary forest, the estimated AGB calculated using the selected proposed allometric equations was $28.53 \mathrm{Mg} \mathrm{ha}^{-1}$ (Table 5), higher than that using formulas by Hashimoto et al. (2004), i.e.,27.91 $\mathrm{Mg} \mathrm{ha}^{-1}$ and Kenzo et al. (2009b), i.e., $27.80 \mathrm{Mg} \mathrm{ha}^{-1}$. On the other hand, it was lower than that using the equations of Rai and Proctor (1986), Yamakura et al. (1986), Brown (1997), Nelson et al. (1999), Chambers et al. (2001), Kettering et al. (2001), Kiyono and Hastaniah (2008), Sierra et al. (2007), Basuki et al. (2009), and Kenzo et al. (2009a), which were $104.58,53.30,50.72,43.70,60.03,31.58$, $42.65,35.30,58.01$, and $41.25 \mathrm{Mg} \mathrm{ha}^{-1}$, respectively (Figure 4.B).
In the 20-year-old secondary forest, the estimate of AGB was $71.75 \mathrm{Mg} \mathrm{ha}^{-1}$ (Table 4), higher than 69.45, $55.58,69.45$, and $54.98 \mathrm{Mg} \mathrm{ha}^{-1}$ of AGB calculated using the formulas of Kettering et al. (2001), Hashimoto et al. (2004), Sierra et al. (2007), and Kenzo et. al. (2009b), respectively. However, it was lower than the estimated AGB calculated using the equations from Rai and Proctor (1986), Yamakura et al. (1986), Brown (1997), Nelson et al. (1999), Chambers et al. (2001), Kiyono and Hastaniah (2008), Basuki et al. (2009), and Kenzo et al. (2009a), i.e., $167.77,119.56,107.22,85.45,128.60,89.94,97.98,76.82$ $\mathrm{Mg} \mathrm{ha}^{-1}$, respectively (Figure 4.C). 
Table 5. Estimation of AGB using various reported relationships for trees (DBH of $\geq 5 \mathrm{~cm}$ ) in the study sites

\begin{tabular}{|c|c|c|c|c|}
\hline \multirow[b]{2}{*}{ Equation } & \multirow[b]{2}{*}{ Author } & \multicolumn{3}{|c|}{ Estimate of AGB $\left(\mathrm{Mg} \mathrm{ha}^{-1}\right)$} \\
\hline & & $\begin{array}{c}\text { 5-year-old } \\
\text { secondary forest }\end{array}$ & $\begin{array}{c}10 \text {-year-old } \\
\text { secondary forest }\end{array}$ & $\begin{array}{c}20 \text {-year-old } \\
\text { secondary forest }\end{array}$ \\
\hline $\ln (\mathrm{AGB})=2.12 \times \ln (\mathrm{DBH})-0.435$ & Rai and Proctor (1986) & 40.61 & 104.58 & 167.77 \\
\hline $\ln (\mathrm{AGB})=2.62 \times \ln (\mathrm{DBH})-2.30$ & Yamakura et al. (1986) & 17.52 & 53.30 & 119.56 \\
\hline $\ln (\mathrm{AGB})=2.53 \times \ln (\mathrm{DBH})-2.13$ & Brown (1997) & 17.24 & 50.72 & 107.22 \\
\hline $\ln (\mathrm{AGB})=2.413 \times \ln (\mathrm{DBH})-1.997$ & Nelson et al. (1999) & 15.48 & 43.70 & 85.45 \\
\hline $\ln (\mathrm{AGB})=2.55 \times \ln (\mathrm{DBH})-2.010$ & Chambers et al. (2001) & 20.26 & 60.03 & 128.60 \\
\hline $\ln (\mathrm{AGB})=2.59 \times \ln (\mathrm{DBH})-2.75$ & Kettering et al. (2001) & 10.50 & 31.58 & 69.45 \\
\hline $\ln (\mathrm{AGB})=2.44 \times \ln (\mathrm{DBH})-2.51$ & Hashimoto et al. (2004) & 9.80 & 27.91 & 55.58 \\
\hline $\ln (\mathrm{AGB})=2.422 \times \ln (\mathrm{DBH})-2.232$ & Sierra et al. (2007) & 12.47 & 35.30 & 69.45 \\
\hline $\mathrm{AGB}=0.1008 \times \mathrm{DBH}^{2.5264}$ & Kiyono and Hastaniah (2008) & 14.52 & 42.65 & 89.94 \\
\hline $\ln (\mathrm{AGB})=2.196 \times \ln (\mathrm{DBH})-1.201$ & Basuki et al. (2009) & 22.03 & 58.01 & 97.98 \\
\hline $\mathrm{AGB}=0.1525 \times \mathrm{DBH}^{2.34}$ & Kenzo et al. (2009a) & 14.98 & 41.25 & 76.82 \\
\hline $\mathrm{AGB}=0.0829 \times \mathrm{DBH}^{2.43}$ & $\begin{array}{l}\text { Kenzo et al. }(2009 b) \\
\text { This study for: }\end{array}$ & 9.79 & 27.80 & 54.98 \\
\hline $\ln (\mathrm{AGB})=2.0859 \times \ln (\mathrm{DBH})-1.75$ & 5 -year-old secondary forest & 10.17 & & \\
\hline $\ln (\mathrm{AGB})=2.2725 \times \ln (\mathrm{DBH})-2.09$ & 10 -year-old secondary forest & & 28.53 & \\
\hline $\ln (\mathrm{AGB})=2.3207 \times \ln (\mathrm{DBH})-1.89$ & 20-year-old secondary forest & & & 71.75 \\
\hline
\end{tabular}

Note: $\mathrm{AGB}=$ above ground biomass ; $\mathrm{DBH}=$ diameter at breast height

Hashimoto et al. (2004), Kenzo et al. (2009b), and Kettering et al. (2001) equations gave similar values of AGB of trees, particularly for the 5- and 10-year-old secondary forests. Hashimoto et al. (2004) developed the equation from the same forest type of secondary forest in East Kalimantan, Indonesia. The low estimated AGB was probably due to the low wood density (0.29 to 0.47$)$. As mentioned earlier, the wood density of the selected trees varied from 0.18 to 0.66 in the 5-year-old secondary forest, 0.25 to 0.66 in the 10 -year-old secondary forest, and 0.24 to 0.74 in the 20 -year-old secondary forest, respectively (Tables 1, 2 and 3). Kenzo et al. (2009b) reported that the allometric equation for mixed species of early succession secondary forest in Niah and Sungai Liku, Sarawak, Malaysia used wood density of 0.35 . The application of Kettering et al. (2001) formula to estimate the AGB obtained a similar value for the 5-year-old secondary forest, lower value for the 10-year-old secondary forest, and higher value for the 20-year-old secondary forest. Ketterings et al. (2001) used the wood densities of 0.35 to 0.91 for the allometric equation for mixed secondary forest with mixed species ( 0.35 to 0.91 in wood density) in Sumatra, Indonesia.

The estimates of AGB in the 5-, 10-, and 20-year-old secondary forests showed overestimation when using the formulas of Basuki et al. (2009), Chambers et al. (2001), Rai and Proctor (1986), and Yamakura, et al. (1986). These four formulas resulted from studies at primary rain forest in Berau Regency of East Kalimantan (Indonesia), Central Amazon, India (Karnataka), and Sebulu of East Kalimantan (Indonesia), respectively. Different characteristics were shown by the primary and secondary forest, in structure, floristic composition, diversity, age distribution, and disturbance intensity. The reported equations for large-size trees of primary forest or secondary forest with long fallow resulted in higher estimates of AGB, when they were applied to the trees of early-successional-stage secondary forest. Likewise, the application of Nelson et al. (1999) and Sierra et al. (2007) equations in the study sites also resulted in overestimates of AGB. These two formulas were reported for mixed species of secondary forest in Central Amazon and Colombia.

The result using equations of mixed species in moist tropical forest (Brown 1997) and secondary forest with mainly Schima wallichii in East Kalimantan, Indonesia (Kiyono and Hastaniah 2005) to calculate AGB showed higher value. This might be due to the higher wood density values of trees in those equations $(0.54$ for Nelson et al.'s equation; 0.40-0.79 for Brown's equation, 0.67 for Kiyono and Hastaniah's equation) than in the selected species in the study sites. The equations of Kenzo et al. (2009a) for mixed species of logged-over tropical rain forest in Sabal and Balai Ringin, Sarawak, Malaysia, resulted in overestimate of AGB when they were used in the study sites. Although, the study sites of Kenzo et al. (2009a) were adjacent to ours, and similar selected species were used to develop allometric equation, but the forest age and land history were different.

The developed allometric equations resulted in intermediate value of AGB among the results of allometric equations for primary forest (Basuki et al. 2009; Yamakura et al. 1986), the secondary forest composed mainly by Schima wallichii (Kiyono and Hastaniah 2008), the loggedover secondary forest (Kenzo et al. 2009a), and the early successional-stage secondary forest (Kenzo et al. 2009b). The higher estimates were obtained using allometric equation for primary tropical forest (Chambers et al. 2001; Rai and Proctor 1986) and secondary forest (Brown 1997; Nelson et al. 1999). Using the equations for mixed secondary forest dominated by Hevea brasiliensis and naturally regenerating trees (Ketterings et al. 2001) and mixed-species (Hashimoto et al. 2004; Sierra et al. 2007) resulted in similar values of TAGB with the developed equation. 

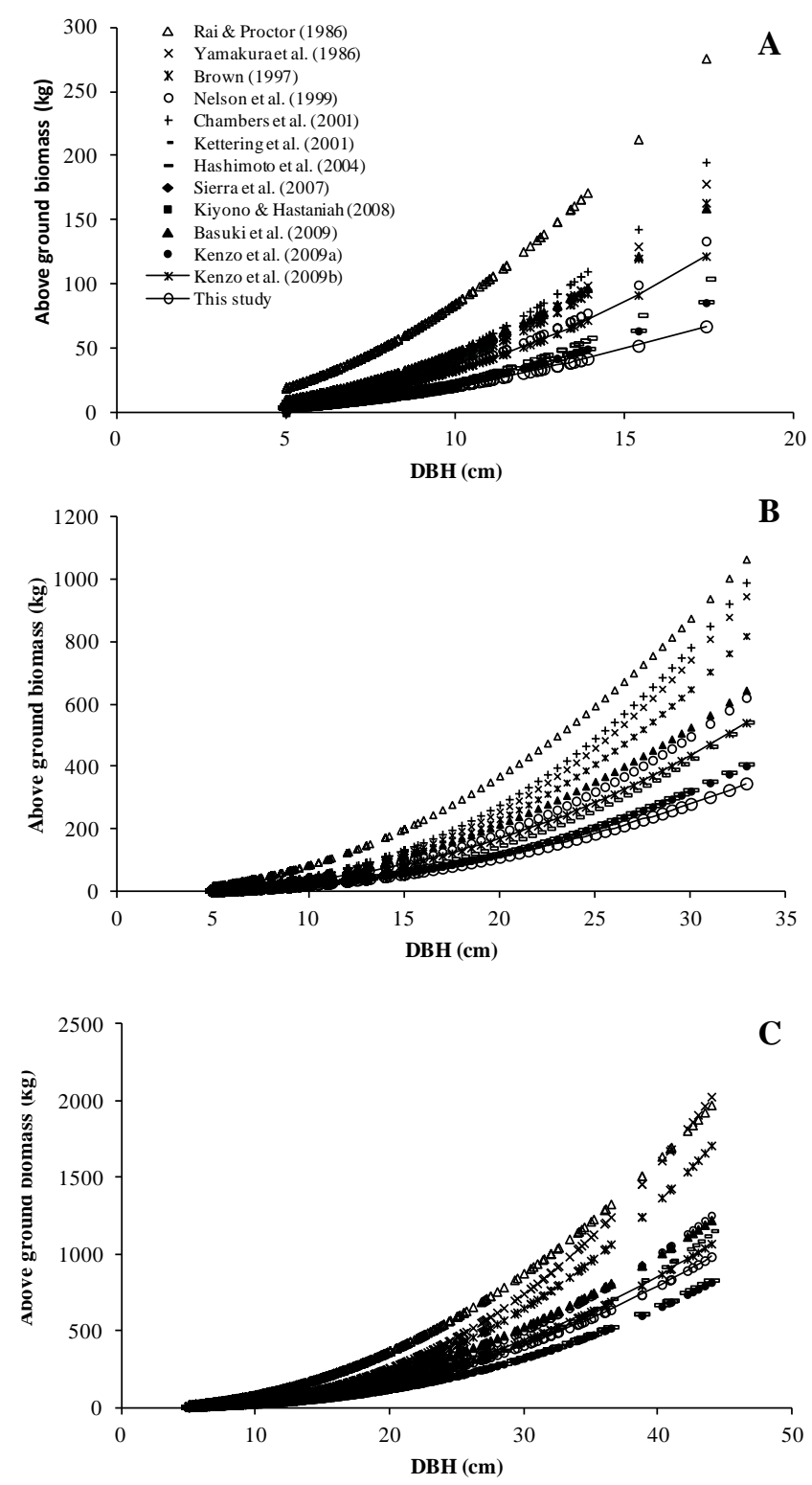

Figure 4. Comparison among various allometric relationships between above ground biomass (AGB) and diameter at breast height (DBH) in the study sites. A. 5-year-old secondary forest, B. 10-year-old secondary forest, C. 20-year-old secondary forest

In conclusion, a specific allometric equation must be developed to estimate the AGB of a specific forest, because the use of inappropriate allometric equations will result in inaccurate estimates of AGB.

\section{ACKNOWLEDGEMENTS}

We express our acknowledgment and appreciation to Hidir Marzuki, Sekudan Tedong, Salim Arip, and Muhd Najib Fardos for their kind assistance during the field survey.

\section{REFERENCES}

Baker TR, Phillips OL, Malhi Y, Almeida S, Arroyo L, Di Fiore A, Killeen TJ, Laurance SG, Laurance WF, Lewis SL, Lloyd J, Monteagudo A, Neill DA, Patińo S, Pitman NCA, Silva N., Martínez RV. 2004. Variation in wood density determines spatial patterns in Amazonian forest biomass. Global Change Biol 10: 545-562.

Basuki TM, Van Laake PE, Skidmore AK, Hussin YA. 2009. Allometric equations for estimating the above-ground biomass in tropical lowland dipterocarp forest. For Ecol Manag 257: 1684-1694.

Bowyer JL, Schmulsky R, Haygreen JG. 2003. Forest Products and Wood Science: An Introduction. Iowa State Press, Iowa.

Brower JE, Zar JH, Von Ende CN. 1990. Field and Laboratory Methods for General Ecology. Wm. C. Brown Publishers, USA.

Brown S. 1997. Estimating Biomass and Biomass Change in Tropical Forest: A Primer. Food and Agriculture Organization of the United Nations, Rome.

Chamber JQ, Santos JD, Ribeiro RJ, Higuchi N. 2001. Tree damage, allometric relationship, and above-ground net primary production in Central Amazon forest. For Ecol Manag 152: 73-84.

Chave J. 2006. Measuring Wood Density for Tropical Forest Trees: A Field Manual. Sixth Framework Programme (2002-2006). Project for the Advancement of Networked Science in Amazonia, France.

Chave J, Andalo C, Brown S, Cairns MA, Chambers JQ, Eamus D, Fölster FH, Fromard F, Higuchi N, Kira T, Lescure, JP, Nelson BW, Ogawa H, Puig H, Riera B, Yamakura T. 2005. Tree allometry and improved estimation of carbon stocks and balance in tropical forests. Oecologia 145: 87-99.

Curtis PS. 2008. Estimating Aboveground Carbon in Live and Standing Dead Trees. In: Hoover CM (ed). Field Measurements for Forest Carbon Monitoring: a Landscape-Scale Approach. Springer, USA.

Hairiah K, Rahayu S. 2007. Pengukuran "Karbon Tersimpan” di Berbagai Macam Penggunaan Lahan. World Agroforestry Centre-ICRAF, Indonesia.

Hairiah K, Sitompul SM, Van Noordwijk M, Palm C. 2001. Methods for Sampling Carbon Stocks Above and Below Ground. ASB Lecture Note 4B. International Centre for Research in Agroforestry, Indonesia.

Hashimotio T, Kojia K, Tange T, Sasaki S. 2000. Changes in carbon storage in fallow forests in the tropical lowlands of Borneo. For Ecol Manag 126: 331-337.

Hashimoto T, Tange T, Masumori M, Yagi H, Sasaki S, Kojima K. 2004. Allometric equations for pioneer tree species and estimation of the aboveground biomass of a tropical secondary forest in East Kalimantan. Tropics 14: 123-130.

Heriansyah I, Heriyanto NM, Siregar CA. 2002. Demonstration Study on Carbon Fixing Forest Management in Indonesia. In: Proceeding Climate Change, Forests and Peatlands in Indonesia (Series 1). Wetlands International, Canada.

Karyati, Ipor IB, Jusoh I, Wasli ME, Seman IA. 2013. Composition and diversity of plant seedlings and saplings at early secondary succession of fallow lands in Sabal, Sarawak. Acta Biologica Malaysiana 2 (3): 85-94.

Karyati K, Ipor IB, Jusoh I, Wasli ME. 2014. Soil properties under various stages of secondary forests at Sarawak, East Malaysia. J Trop For Environ 4 (1): 28-39.

Karyati, Ipor IB, Jusoh I, Wasli ME. 2018. Tree stand floristic dynamics in secondary forests of different ages in Sarawak, Malaysia. Biodiversitas 19 (3): 687-693.

Kawahara T, Kanazawa Y, Sakurai S. 1981. Biomass and net production of man-made forests in the Philippines. J Japanese For Soc 63: 320327.

Kendawang JJ, Ninomiya I, Tanaka K, Ozawa T, Hattori D, Tanaka S, Sakurai K. 2007. Effects of burning strength in shifting cultivation on the early stage of secondary succession in Sarawak, Malaysia. Tropics 16: 309-321.

Kenzo T, Furutani R, Hattori D, Kendawang JJ, Tanaka S, Sakurai K, Ninomiya I. 2009a. Allometric equations for accurate estimation of above-ground biomass in logged-over tropical rainforests in Sarawak, Malaysia. J For Res 14 (6): 365-372.

Kenzo T, Ichie T, Hattori D, Itioka T, Handa C, Ohkubo T, Kendawang JJ, Nakamura M, Sakaguchi M, Takahashi N, Okamoto M, TanakaOda A, Sakurai K, Ninomiya I. 2009b. Development of allometric relationships for accurate estimation of above- and below-ground 
biomass in tropical secondary forests in Sarawak, Malaysia. J Trop Ecol 25: 371-386.

Ketterings QM, Coe R, Van Noordwijk M, Ambagau Y, Palm CA. 2001. Reducing uncertainty in the use of allometric biomass equations for predicting above-ground tree biomass in mixed secondary forests. For Ecol Manag 146:199-209.

Kiyono Y, Hastaniah. 2005. Pattern of slash-and-burn land use and their effects on forest succession. swidden-land forests in Borneo. Bull For For Prod Res Inst 4: 259-282.

MacDicken K. 1997. A Guide to Monitoring Carbon Storage in Forestry and Agroforestry Projects. Winrock International, USA.

Marklund LG. 1986. Wood Biomass - A Potential Resource for Energy and Industry. Food and Agriculture Organization of the United Nations, Bangkok.

Ministry of Forestry Indonesia. 2011. Development of Allometric Equations for Estimating Forest Carbon Stocks Based on Field Measurement (Ground-Based Forest Carbon Accounting). Centre for Standardization and Environment, Ministry of Forestry, Indonesia.

Nelson BW, Mesquita R, Pereira JLG, De Souza SGA, Batista T, Couto LB. 1999. Allometric regressions for improved estimate of secondary forest biomass in the Central Amazon. For Ecol Manag 117:149-167.
Nogueira EM, Nelson BW, Fearnside PM. 2005. Wood density in dense forest in Central Amazonia, Brazil. For Ecol Manag 208: 261-286.

Nogueira EM, Fearnside PM, Nelson BW, França MB. 2007. wood density in forests of Brazil's 'arc of deforestation': Implications for biomass and flux of carbon from land-use change in Amazonia. For Ecol Manag 248: 119-135.

Perera GAD. 2001. The secondary forest situation in Sri Lanka: A review. J Trop For Sci 13 (4): 768-785

Rai SN, Proctor J. 1986. Ecological studies on four rainforest in Karnataka India. J Ecol 76:938-958.

Ruiz-Jaen MC, Potvin C. 2010. Tree diversity explains variation in ecosystem function in a neotropical forest in Panama. Biotropica 42 (6): 638-646.

Sierra CA, Del Valle JI, Orrego SA, Moreno FH, Harmon MA, Zapata M, Colorado GJ, Herrera MA, Lara W, Restrepo DE, Berrouet LM, Loaiza LM, Benjumea JF. 2007. Total carbon stocks in a tropical forest landscape of the Porce Region, Colombia. For Ecol Manag 243: 299-309.

Yamakura T, Hagihara A, Sukardjo S, Ogawa H. 1986b. Aboveground biomass of tropical rain forest stands in Indonesian Borneo. Vegetatio 68: 71-82. 